\title{
Asaia, a versatile acetic acid bacterial symbiont, capable of cross-colonizing insects of phylogenetically distant genera and orders
}

Elena Crotti, ${ }^{1 \S}$ Claudia Damiani, ${ }^{2 \S}$ Massimo Pajoro, ${ }^{3 \S}$ Elena Gonella, ${ }^{3 \S}$ Aurora Rizzi, ${ }^{1}$ Irene Ricci, ${ }^{2}$ Ilaria Negri, ${ }^{3}$ Patrizia Scuppa, ${ }^{2}$ Paolo Rossi, ${ }^{2}$ Patrizia Ballarini, ${ }^{4}$ Noura Raddadi, ${ }^{1,3 \dagger}$ Massimo Marzorati, ${ }^{1 \ddagger}$ Luciano Sacchi, ${ }^{5}$

Emanuela Clementi, ${ }^{5}$ Marco Genchi, ${ }^{5}$ Mauro Mandrioli, ${ }^{6}$ Claudio Bandi, ${ }^{7}$ Guido Favia, ${ }^{2}$ Alberto Alma ${ }^{3}$ and Daniele Daffonchio ${ }^{1 *}$ ${ }^{1}$ Dipartimento di Scienze e Tecnologie Alimentari e Microbiologiche, Università degli Studi di Milano, 20133 Milan, Italy.

${ }^{2}$ Dipartimento di Medicina Sperimentale e Sanità

Pubblica, Università degli Studi di Camerino, 62032 Camerino, Italy.

${ }^{3}$ Dipartimento di Valorizzazione e Protezione delle Risorse Agroforestali, Università degli Studi di Torino, 10095 Turin, Italy.

${ }^{4}$ Dipartimento di Biologia Molecolare, Cellulare ed Animale, Università degli Studi di Camerino, 62032 Camerino, Italy.

${ }^{5}$ Dipartimento di Biologia Animale, Università degli Studi di Pavia, 27100 Pavia, Italy.

${ }^{6}$ Dipartimento di Biologia Animale, Università degli Studi di Modena e Reggio Emilia, 41125 Modena, Italy.

${ }^{7}$ Dipartimento di Patologia Animale, Igiene e Sanità Pubblica Veterinaria, Università degli Studi di Milano, 20133 Milan, Italy.

\section{Summary}

Bacterial symbionts of insects have been proposed for blocking transmission of vector-borne pathogens. However, in many vector models the ecology of symbionts and their capability of cross-colonizing different hosts, an important feature in the symbiotic control approach, is poorly known. Here we show that the acetic acid bacterium Asaia, previously found in the

Received 22 December, 2008; accepted 31 July, 2009. *For correspondence. E-mail daniele.daffonchio@ unimi.it; Tel. (+39) 250319117; Fax (+39) 250319238. Present addresses: 'Dipartimento di Valorizzazione e Protezione delle Risorse Agroforestali, Università degli Studi di Torino, 10095 Turin, Italy; ' Laboratory for Microbial Ecology and Technology (LabMET), Ghent University, B9000 Ghent, Belgium. ${ }^{\S}$ These authors contributed equally to this work. malaria mosquito vector Anopheles stephensi, is also present in, and capable of cross-colonizing other sugar-feeding insects of phylogenetically distant genera and orders. PCR, real-time PCR and in situ hybridization experiments showed Asaia in the body of the mosquito Aedes aegypti and the leafhopper Scaphoideus titanus, vectors of human viruses and a grapevine phytoplasma respectively. Crosscolonization patterns of the body of Ae. aegypti, An. stephensi and S. titanus have been documented with Asaia strains isolated from An. stephensi or Ae. aegypti, and labelled with plasmid- or chromosome-encoded fluorescent proteins (Gfp and DsRed respectively). Fluorescence and confocal microscopy showed that Asaia, administered with the sugar meal, efficiently colonized guts, male and female reproductive systems and the salivary glands. The ability in cross-colonizing insects of phylogenetically distant orders indicated that Asaia adopts body invasion mechanisms independent from host-specific biological characteristics. This versatility is an important property for the development of symbiont-based control of different vector-borne diseases.

\section{Introduction}

Microorganisms play crucial roles in the biology and life cycle of most arthropod species, affecting nutrition, development, reproduction, immunity, defence against natural enemies and speciation (Dale and Moran, 2006; Moran, 2006; Feldhaar and Gross, 2009). Animal symbioses are categorized according to the extent of dependence between the host and the symbiont, which generally depends on evolutionary antiquity of the symbiosis. While obligate primary symbionts are essential for the host survival and/or reproduction, secondary symbionts are facultative and thought to be of more recent acquisition, even though they can contribute to the fitness of the host, e.g. conferring resistance to parasites (Dale and Moran, 2006). Most primary symbionts are vertically transmitted to the progeny with a process starting at early stages of oogenesis or embryogenesis. Vertical transmission is common also in secondary symbionts, but they can also colonize novel hosts through horizontal transmission 
among host individuals belonging to the same or different species (Dale and Moran, 2006).

Representatives from the group of the acetic acid bacteria (AAB), e.g. Acetobacter, Gluconacetobacter and Gluconobacter, have recently been demonstrated to be naturally associated, not only with plants (Kommanee et al., 2008), but also with insects, such as the fruit fly Drosophila melanogaster (Corby-Harris et al., 2007; Cox and Gilmore, 2007; Ren et al., 2007; Ryu et al., 2008), the olive fly Bactrocera oleae (Kounatidis et al., 2009), the honeybee Apis mellifera (Jeyaprakash et al., 2003; Mohr and Tebbe, 2006; Babendreier et al., 2007) and the pink sugarcane mealybug Saccharococcus sacchari (Ashbolt and Inkerman, 1990). Cox and Gilmore (2007) found that in D. melanogaster, Acetobacter was one of the most abundant genera accounting for $29 \%$ of all phylotypes. The detection of AAB in the above insects, all having plant materials and sugar-rich matrices as food sources, suggests that these bacteria might play roles in food exploitation.

Among $\mathrm{AAB}$, the genus Asaia differentiates because it does not (or weakly) oxidize ethanol to acetic acid. Besides tropical plants, where it was originally isolated (Yamada et al., 2000; Katsura et al., 2001; Yukphan et al., 2004; Malimas et al., 2008), Asaia has thus far been found associated with few insect species. These include the hemipteran Scaphoideus titanus, the leafhopper vector of the phytoplasma causing Flavescence Dorée, a severe disease of grapevine (Marzorati et al., 2006), and three mosquito vectors of malaria, Anopheles stephensi, Anopheles maculipennis and Anopheles gambiae. Asaia has been found stably associated with larvae and adults of An. stephensi, dominating the microbiota of the mosquito (Favia et al., 2007). The distribution of Asaia in the body of $A n$. stephensi has been investigated by the use of a strain, previously isolated from the mosquito, after genetic modification to express a green fluorescent protein (Gfp). The Gfp-tagged strain efficiently colonized the gut, salivary glands and male and female reproductive organs. It is noteworthy that Asaia, after assumption with a sugar-based diet by females, was detected in the gut and then in the salivary glands of the insect, crucial organs for the development of the cycle of the malaria parasites Plasmodium spp. (Favia et al., 2007). By using fluorescent strains it was shown that, in An. stephensi, Asaia is vertically transmitted from the mother to offspring (Favia et al., 2007), but also undergoes paternal transmission to the progeny, by the way of venereal transfer from male to female during mating (Damiani et al., 2008).

The efficient capacity of Asaia of colonizing adults and larvae of An. stephensi by the way of multiple transmission routes (horizontal acquisition through feeding, venereal transmission from male to female and maternal transmission to offspring), and the discovery of this bacterium in other insect vectors (i.e. other Anopheles species and $S$. titanus) raise the question of whether, and to which extent, this bacterium can cross-colonize different insect hosts. This capacity would allow investigating the basis of host-symbiont specific interaction and could be very promising towards the development of Asaiabased symbiotic control approaches to block parasite transmission by insect vectors. A high specificity of the host-symbiont interaction characterizes primary symbionts that are vertically transmitted to the progeny but are not cultivable. This hampers their use for symbiotic control. On the other hand, secondary symbionts include culturable microorganisms that are transmitted both vertically and horizontally. For symbiotic control applications, multiple transmission routes and broad host range carry some potential risks regarding biosafety of the biocontrol symbiotic microorganism, as well as opportunities for a successful disease transmission control. The more the transmission routes, the higher the chances of success are. In addition, if a symbiont is present in many different hosts, the chances of environmental spread and horizontal transmission to the target species are higher. In this work, the capacity of Asaia symbionts to cross-colonize insects of phylogenetically distant orders, like Diptera and Hemiptera, has been evaluated by using as models An. stephensi and Aedes aegypti (Diptera) and S. titanus (Hemiptera). We chose these species according to their evolutionary distance, one, Ae. aegypty, phylogenetically close to An. stephensi, the other, S. titanus, much more distant. The presence of Asaia and the ability to crosscolonize organs and tissues of these insects were assessed by in situ hybridization, quantitative real-time PCR and cultivation, and by in vivo studies with Asaia strains labelled with Gfp or DsRed fluorescent proteins after isolation from An. stephensi (Favia et al., 2007) and Ae. aegypti.

\section{Results}

Detection of Asaia in Ae. aegypti and S. titanus

Electron microscopy observations on midgut sections of Ae. aegypti and S. titanus individuals (Fig. 1) allowed us to observe bacterial cells resembling Asaia in both insects. Figure $1 \mathrm{~A}$ shows a portion of the midgut of $A e$. aegypti filled with bacteria, embedded in an extracellular matrix, whose morphology is very similar to that already reported for Asaia in An. stephensi (Favia et al., 2007). Two morphological signatures, i.e. a bright filamentous nucleoid region and electron-dense cytoplasm microinclusions resembling enterosomes, organelles rarely described in bacterial cells (Schaechter et al., 2006), were previously recognized and described for Asaia cells (Favia et al., 2007). The finding of these signatures in bacterial cells of midgut sections of Ae. aegypti and 

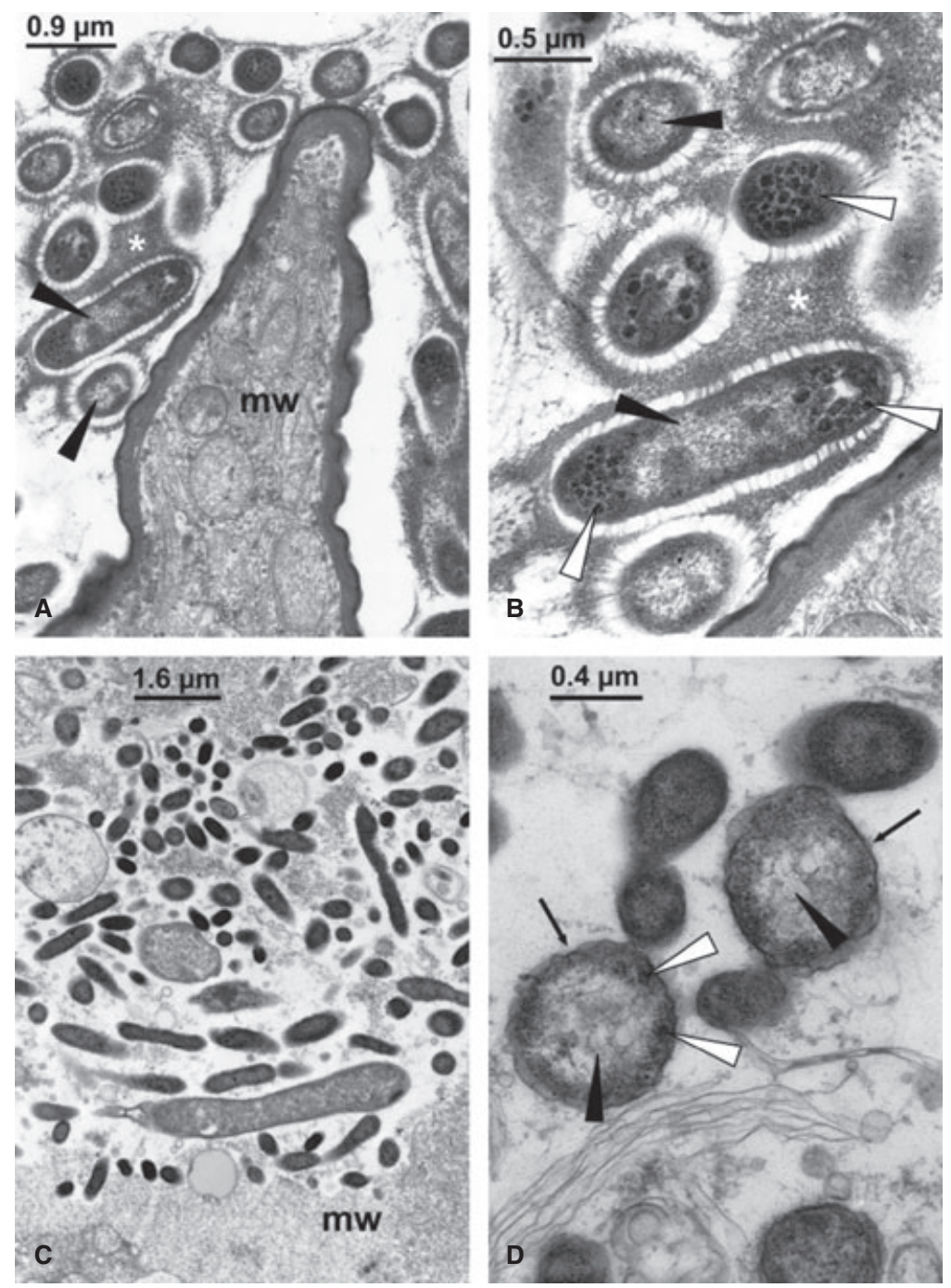

Fig. 1. Transmission electron microscopy micrographs of the midgut of Ae. aegypti and S. titanus.

A. Midgut of Ae. aegypti filled with bacteria that can be interpreted as belonging to the genus Asaia; the bacteria are embedded within an extracellular matrix (asterisk). Bright filamentous nucleoid regions are indicated by black arrowheads; mw: midgut wall.

$B$. Detail of $(A)$; note the presence of the nucleoid region (black arrowheads) and the enterosomes in the bacterial cytoplasm (white arrowheads) and extracellular matrix (asterisk).

C. Midgut of $S$. titanus filled with a highly polymorphic bacterial flora; mw: midgut wall. D. Detail of the midgut lumen of $S$. titanus showing two bacteria (indicated with arrows) that can be interpreted as belonging to the genus Asaia. Bright nucleoid regions are indicated by black arrowheads, while enterosomes are indicated with white arrowheads.
S. titanus individuals suggested the presence of Asaia. Thus, according to their ultrastructure, the bacteria in $A e$. aegypti could be attributed to the genus Asaia (Fig. 1B). In $S$. titanus, the midgut hosts a polymorphic bacterial flora (Fig. 1C). Some of the bacteria observed in the gut of $S$. titanus also presented a bright filamentous nucleoid region (Fig. 1D), resembling that described for Asaia.

In order to verify the presence of Asaia sp. in Ae. aegypti, DNA was extracted from individual mosquitoes at different life cycle stages and PCR was performed using Asaia-specific primers. A total of 150 individuals, 30 for each of five successive generations, were analysed. The expected bands were detected in all the tested individuals, both in the preimago stages, i.e. larvae and pupae (40 individuals each, respectively), and adults (35 males and 35 females). To confirm the identity of the amplified products, 35 were randomly chosen for sequencing. All of them showed nucleotide identity higher than $99 \%$ with Asaia bogorensis and Asaia siamensis. Asaia-specific
PCRs were also carried out on mosquito male and female guts (respectively from 10 and 15 individuals), male and female reproductive organs and salivary glands (from 20 individuals each). All of the samples were positive for Asaia and the identity of the amplified 16S rRNA gene fragments was confirmed as Asaia by sequencing 12 randomly chosen amplicons.

Forty adults of $S$. titanus (males and females, 20 each) were analysed for the presence of Asaia in the body following the same approach used for Ae. aegypti, resulting in 30 positive samples (14 males and 16 females respectively). Among the positive samples, 11 individuals (5 males and 6 females) were analyed by quantitative PCR in order to measure the relative abundance of Asaia within the microbiota in the body of $S$. titanus. Real-time quantitative PCRs targeting specifically Asaia sp. or total bacteria were used to determine the Asaia and the total bacterial 16S rRNA gene copies. The two values were then used to calculate the Asaia to total bacteria $16 \mathrm{~S}$ 


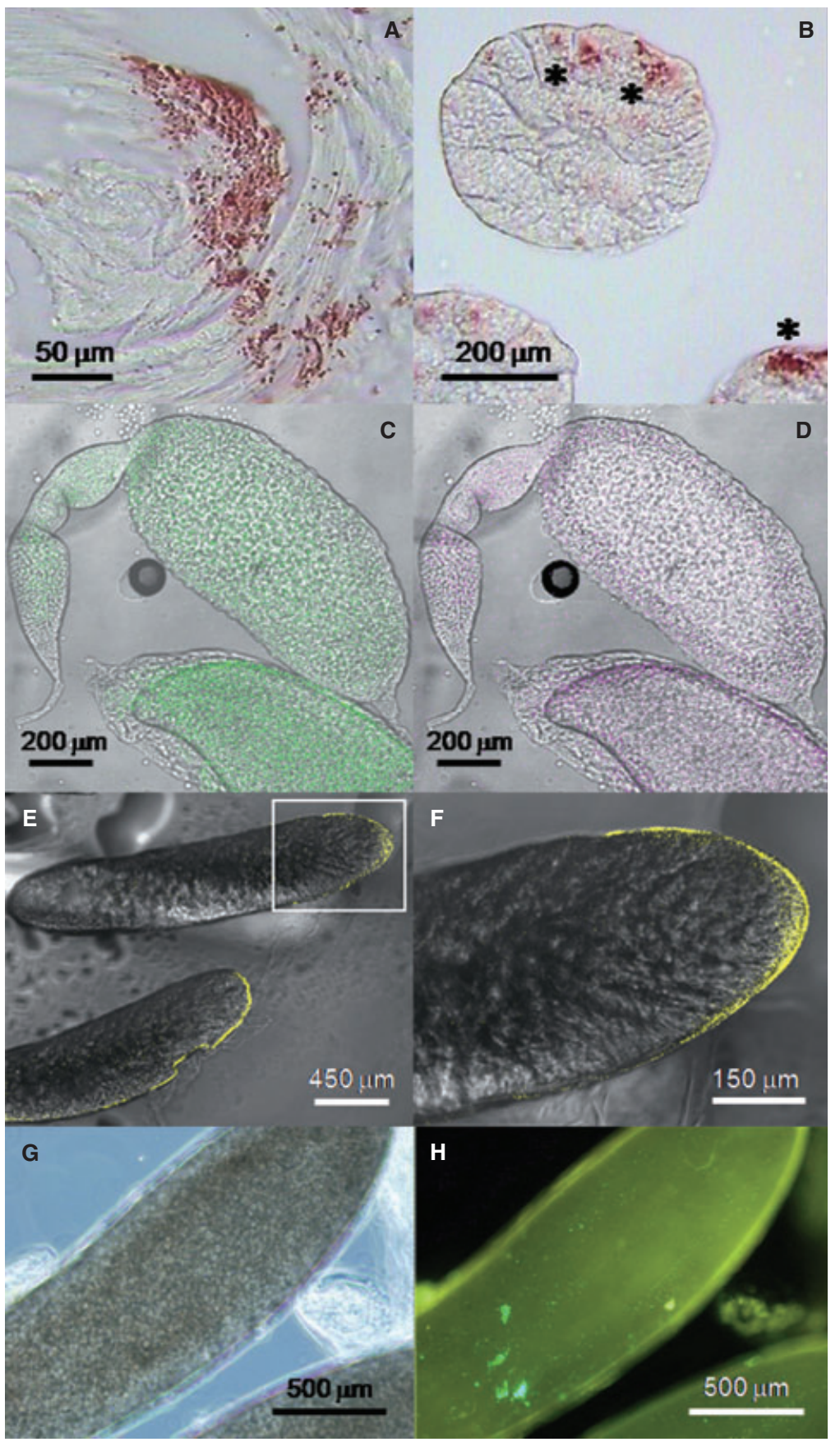

Fig. 2. Detection of naturally occurring Asaia sp. in S. titanus by ISH and FISH and colonization of the ovarian eggs of $S$. titanus by Asaia SF2.1(Gfp). Signals obtained by ISH with Asaia-specific probes (Asaia1 and Asaia2, 5'-labelled with digoxigenin) were observed in the spermatic bundles (A) and in the Malpighian tubules (asterisks) (B). Ovarian eggs, investigated by FISH, are shown in $(\mathrm{C}-\mathrm{F})$. The universal bacterial probe EUB388, labelled with FITC (green colour), was used for total bacteria visualization in (C). Specific Asaia-probes, Asaia1 and Asaia2 probes $5^{\prime}$ end labelled with the fluorochrome Cy5, showed the presence of Asaia cells all around the immature eggs (D). Ovarian eggs in a more advanced stage of maturation hybridized with the Cy3-labelled Asaia-specific probes Asaia1 and Asaia2 show high concentrations of Asaia cells around the egg apical regions (E and F). Single cells and microcolonies of Gfp-labelled Asaia can be observed on the surface of mature ovarian eggs $(H)$. The correspondent phase contrast image is presented in $(G)$.

rRNA gene copy ratio within the insects. The number of Asaia 16S rRNA gene copies per individual covered a range from $1.0 \times 10^{3}$ to $7.3 \times 10^{8}$, with an average value of $9.4( \pm 6.9) \times 10^{7}$. The number of total bacteria 16S rRNA gene copies per individual covered a range from $1.4 \times 10^{6}$ to $1.9 \times 10^{9}$, with an average value of $8.3( \pm 3.9) \times 10^{8}$. Assuming that about four rRNA gene copies per cell are present in Asaia, as observed in other AABs (http:// ribosome.mmg.msu.edu/rrndb/index.php, Klappenbach et al., 2001), we could estimate an average of $2.3 \times 10^{7}$
Asaia cells associated with an individual of S. titanus. The average Asaia to Bacteria 16S rRNA gene copy ratio (ABR) was 0.049 , indicating that Asaia 16S rRNA gene copies constituted a mean of $4.9 \%$ of the total bacterial 16S rRNA gene copies.

In order to investigate the presence and the localization of Asaia in different leafhopper organs, in situ hybridization (ISH) and fluorescent ISH (FISH) were performed. Asaia was detected by ISH in testicles, intermixed within the spermatic bundles (Fig. 2A) and in the Malpighian 

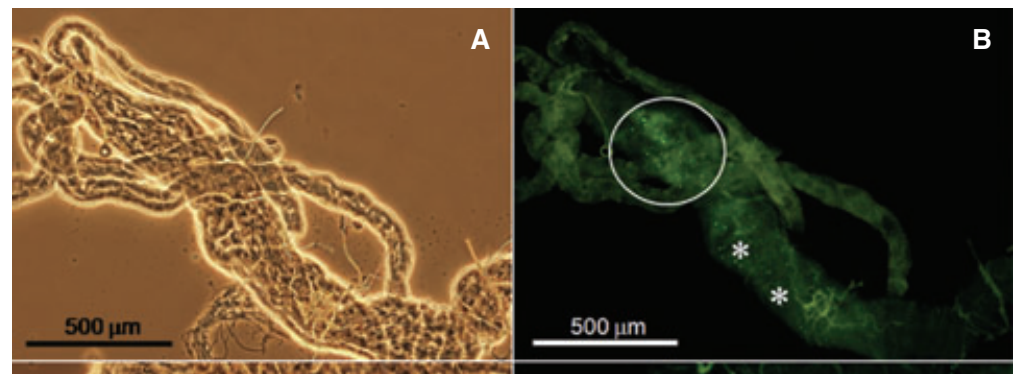

Fig. 3. Colonization of the adult gut of Ae. aegypti by Asaia SF2.1(Gfp). Phase contrast $(A$ and $C$ ) and fluorescence ( $B$ and $D)$ microscope images of guts of a male (A and $B$ ) and a female (C, D), fed with a sucrose solution containing Asaia SF2.1(Gfp). Clusters (circles) and spots (asterisks) of bright fluorescent recombinant Asaia cells are visible by fluorescence microscopy.

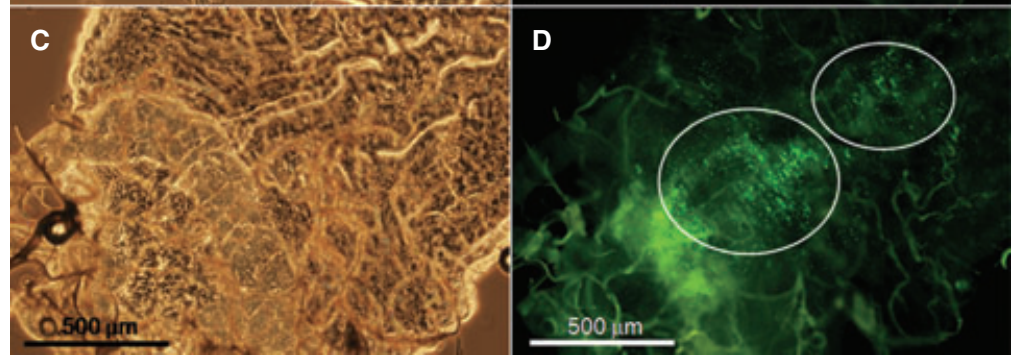

nucleotide identity higher than $99 \%$ with $A$. bogorensis

tubules (Fig. 2B). Leafhopper ovarian eggs were investigated by FISH. Figure $2 \mathrm{C}-\mathrm{D}$ show ovarioles and an immature egg hybridized with the universal bacterial probe EUB338 (green colour) or with the Asaia-specific probes (pink colour) respectively. The immature eggs were covered by bacteria most of which corresponded to Asaia. Asaia-specific probe was also used on more mature ovarian eggs, detecting bright signals all around the egg with particular concentration on the egg edge reads apical regions (Fig. $2 \mathrm{E}-\mathrm{F}$ ). Hence, during the egg maturation Asaia appeared to pass from a dispersed localization scattered on the entire egg surface to a more peripheral localization with an apparent higher cell concentration in the egg apical regions. In all the ISH and FISH experiments no signals were observed in tissues treated with RNase or in the absence of the probe.

\section{Isolation of Asaia from Ae. aegypti}

Asaia sp. from Ae. aegypti individuals was successfully isolated in pure culture by using a liquid enrichment medium at $\mathrm{pH} 3.5$ followed by plating in a carbonatecontaining solid medium (Yamada et al., 2000). The enrichment medium allowed the elimination of those microorganisms not tolerating low $\mathrm{pH}$, while the following plating on $\mathrm{CaCO}_{3}$-containing medium permitted the isolation of acid-producing bacteria capable of creating $\mathrm{CaCO}_{3}$ dissolution haloes around the colonies. Twentythree pink-pigmented, shiny and smooth colonies isolated from four adult mosquitoes, two females and two males, were selected for further analyses. All the collected strains were positive when subjected to Asaiaspecific PCR. 16S rRNA gene sequencing of three randomly selected colonies, named AE5.2, AE6.5 and $\mathrm{AE} 10.8$, confirmed their identification as Asaia sp. with a or A. siamensis.

The same approach was adopted for isolation of Asaia sp. from nymphs and adults of $S$. titanus. However, no Asaia strains could be isolated, neither when sorbitol was substituted with glucose, sucrose or mannitol as carbon source.

\section{Colonization patterns of Asaia in mosquitoes and leafhoppers}

The pattern of colonization of the host body by Asaia was investigated in Ae. aegypti, An. stephensi and S. titanus individuals. Mosquitoes and leafhoppers were fed with sucrose solutions containing fluorescent Asaia cells: both species of mosquitoes were fed with Asaia cells expressing plasmid-encoded Gfp while leafhoppers were fed with Asaia cells expressing either plasmid-encoded Gfp or chromosome-encoded DsRed. Asaia strains SF2.1(Gfp) and AE10.8(Gfp), originally isolated from An. stephensi (Favia et al., 2007) and Ae. aegypti, respectively, were provided to mosquitoes together with kanamycin in order to avoid the rapid loss of the plasmid carrying the Gfp. Indeed, experiments conducted in vitro showed that, without antibiotic supplementation, the percentage of Asaia cells retaining plasmid pHM2-Gfp halved after 24 generations, dropping to $21 \%$ after 36 generations. To overcome the biasing effect of the antibiotic selection, Asaia strain SF2.1(DsRed), chromosomally tagged with a DsRed coding cassette and thus indefinitely retaining fluorescence, was prepared. This strain was provided to insects in the same conditions of strains SF2.1(Gfp) or AE10.8(Gfp) but without antibiotic in the medium.

The colonization patterns of Asaia labelled with optical markers in the body parts of mosquitoes and leafhopper 


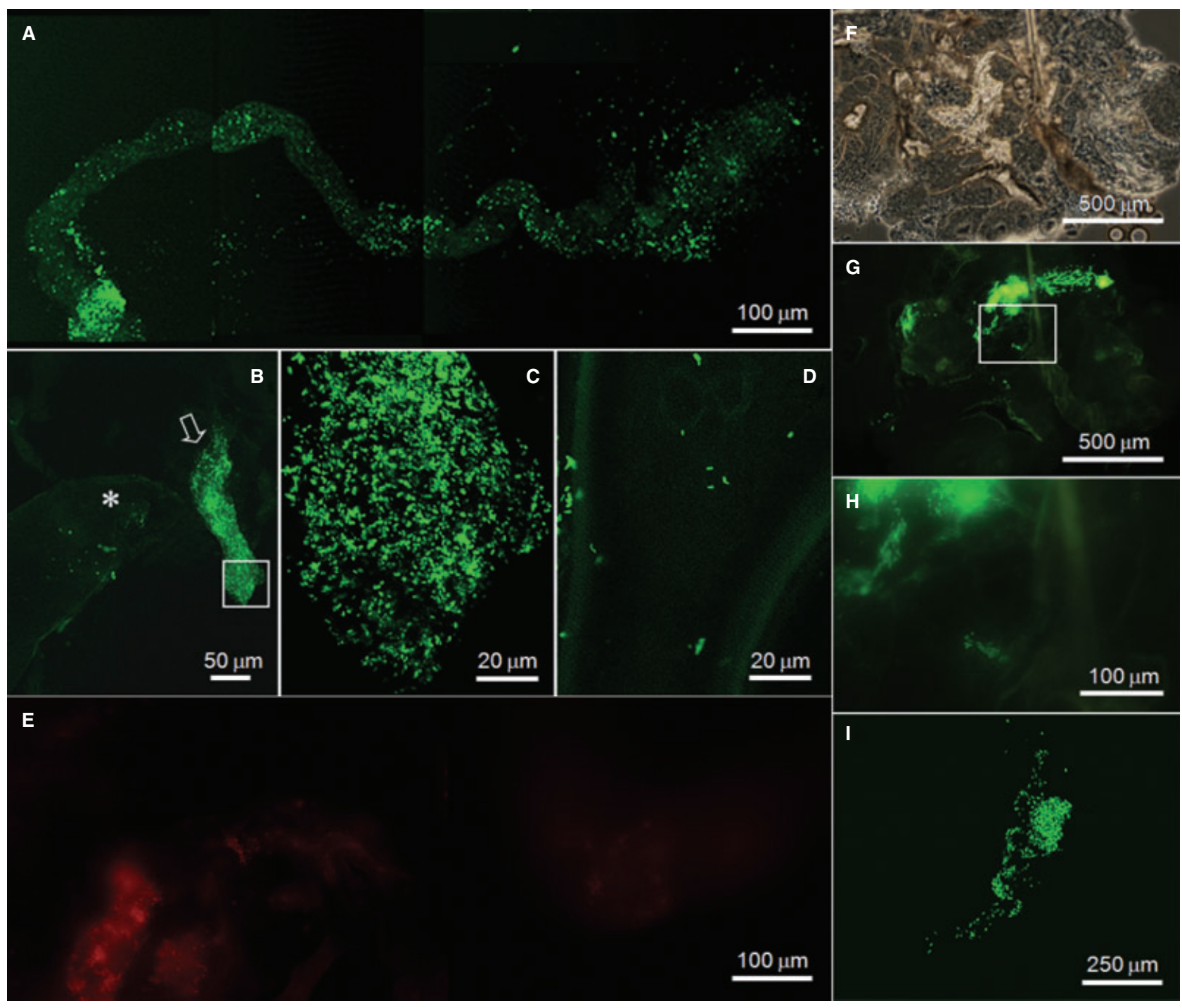

Fig. 4. Colonization of $S$. titanus by Asaia SF2.1(Gfp) (A-D and F-I) and SF2.1(DsRed) (E), documented by confocal laser scanning (A-D, I) and fluorescence $(E, G, H)$ microscopy.

A. Reconstructed image of a female leafhopper gut obtained by overlapping four successive sections.

B. View of the ovary duct (arrow) next to an egg (asterisk). The ovary duct is fully colonized by the recombinant strain.

C. Magnification of a portion of the ovary duct (inset in panel B) in which large microcolonies of Asaia can be observed.

D. Observation of Asaia SF2.1(Gfp) on the surface of an immature egg.

E. Colonization of $S$. titanus gut by Asaia SF2.1(DsRed).

G. Salivary glands showing a massive colonization of Asaia SF2.1(Gfp). The corresponding phase contrast image is shown in (F).

$\mathrm{H}$. Magnification of a portion of the salivary gland (inset in panel $\mathrm{G}$ ) in which large microcolonies of Asaia can be observed.

I. Colonization of the deferent duct in the male reproductive system of S. titanus by Asaia SF2.1(Gfp).

are shown in Figs 3 and 4. The Gfp-tagged strains SF2.1(Gfp) and AE10.8(Gfp) were used for the experiments of cross-colonization of Ae. aegypti (Fig. 3) and An. stephensi (data not shown; Favia et al., 2007) respectively. Both strains were able to efficiently cross-colonize the guts of males and females of both mosquitoes. Most of the individuals examined (45 out of $77,58.4 \%$ in $A e$. aegypti and 36 out of $36,100 \%$ in An. stephensi) showed fluorescent cells and microcolonies in the gut. In both males (Fig. 3A and B) and females (Fig. 3C and D), detectable colonization first appeared already after $12 \mathrm{~h}$ from feeding and was recorded after 24,48 or $72 \mathrm{~h}$ from the bacterial exposure. Indeed, massive colonization of the gut, intended as the detection of large microcolonies in different portions of the entire gut, was recorded in Ae. aegypti as well as in An. stephensi already after 24 or $48 \mathrm{~h}$ post exposure. Similarly, the analysis was also extended to the reproductive organs and salivary glands of individuals of the two mosquito species. Asaia colonization in the male genital system was achieved in 3 out of

(C) 2009 Society for Applied Microbiology and Blackwell Publishing Ltd, Environmental Microbiology, 11, 3252-3264 
$37(8.1 \%)$ Ae. aegypti specimens and in 6 out of $12(50 \%)$ An. stephensi specimens. Concerning female organs, colonization was detected in 1 out of $39(2.6 \%)$ individuals of Ae. aegypti and 7 out of 19 (36.8\%) An. stephensi. In salivary glands, colonization was revealed in 3 out of 27 (11.1\%) Ae. aegypti specimens and in 5 out of 17 (29.4\%) An. stephensi specimens.

Colonization of the body of adult $S$. titanus by Asaia supplemented through the diet was studied by using Gfp and DsRed fluorescent derivatives of strain SF2.1. In Fig. $4 \mathrm{~A}$, a reconstruction of $S$. titanus intestine by overlapping four sections of the organ acquired by confocal laser scanning microscopy (CLSM) shows a massive colonization of Asaia along all the gut. Colonization of the gut was observed in $74 \%$ and $62 \%$ of individuals, respectively, exposed to Gfp- or DsRed-labelled Asaia cells (39 of 53 and 34 of 55 individuals respectively). In Fig. 4F-H, a typical colonization pattern of the salivary glands of S. titanus is shown. In total, $32 \%$ and $29 \%$ of individuals exposed, respectively, to Gfp- or DsRed-labelled Asaia cells (12 of 38 and 10 of 34 individuals respectively) showed colonization of the salivary glands by the bacterium. Massive colonization with fluorescent bacterial cells was detected in the female gonoduct (Fig. 4B). Higher magnification of Gifp signals made possible to appreciate the homogeneous distribution of Asaia SF2.1(Gfp) in the ovary duct and accumulations of Gfp-positive microcolonies (Fig. 4C). The presence of structured microcolonies, with cells undergoing division, indicates an active bacterial growth. A similar colonization pattern was observed when DsRed-labelled bacteria were used in the diet. In total, $60 \%$ and $57 \%$ of individuals exposed, respectively, to Gfp- or DsRed-labelled Asaia cells (25 out of 42 and 28 out of 49 respectively) resulted positive to colonization of the female reproductive system. Fluorescent cells were also able to reach the immature eggs contained in the ovaries. In Fig. 4D and in Fig $2 \mathrm{G}$ and $\mathrm{H}$ fluorescent cells are observed all around an immature egg in an ovary of $S$. titanus. Figure 4E shows a leafhopper gut recolonized by DsRed-tagged Asaia. Similarly to Ae. aegypti, Asaia SF2.1(Gfp) demonstrated to be capable of colonizing the male reproductive apparatus of $S$. titanus. Figure $4 \mathrm{I}$ shows the massive colonization of the male deferent ducts.

The specificity of the colonization of the insect body by Asaia was assessed by feeding the insects with a DsRedlabelled Escherichia coli or a Gfp-labelled Pseudomonas putida alone or in mixture with Asaia labelled with the Gfp or the DsRed fluorescent proteins. In particular, the mosquitoes were fed with mixtures of Gfp-labelled Asaia and a DH5 $\alpha$ pKan(DsRed) E. coli. After mosquito dissection only green signals of Asaia cells were detected, while red signals were never found in the different organs and tissues examined. In the case of $S$. titanus, experiments for assessing the specificity of colonization of the host body were performed by using: (i) a mixture of $P$. putida KT2442 (gfp Rif'), carrying the gene encoding for the fluorescent protein on the bacterial chromosome, with Asaia SF2.1(DsRed); (ii) a mixture of E. coli DH5o pKan(DsRed) with Asaia SF2.1(Gfp); (iii) P. putida KT2442 (gfp Rif') alone; and (iv) E. coli DH5 $\alpha$ pKan(DsRed) alone. The green or red signals of $P$. putida KT2442 (gfp Rif') or E. coli DH5 $\alpha$ pKan(DsRed) were never recorded by fluorescence or CLS microscopy of the insect dissections. These experiments confirmed a specific ability of Asaia to colonize the bodies of the two insect hosts.

\section{Discussion}

Symbiotic control strategies rely on the exploitation of symbiotic microorganisms with the aim of interfering with the transmission of diseases vectored by insects or to impair the insect biological cycle. Ideally, primary symbionts would be the most interesting for developing a symbiotic control approach since they are obligate symbionts and established a very strict dependence on the host. They are generally restricted to a given specific host. The efficient vertical transmission of these symbionts would guarantee their rapid spread. However, due to a long co-evolution with the host they present several characteristics, including genome reduction, the loss of some essential cellular functions that determine uncultivability. As a consequence, they are not tractable for developing an effective symbiotic control approach. On the contrary, secondary or facultative symbionts can retain the ability to grow in cell-free media, being tractable for microbiological and genetic characterization and manipulation, essential steps for the development of symbiotic control approaches. The capability of growing outside the insect host allows these microorganisms to grow in environmental niches other than the host body, and to be horizontally acquired by the host from the environment. The capability of colonizing different hosts is also interesting, since it would allow to the symbiont multiple ways of environmental release. These capabilities are important ecological traits for the efficacy of symbiotic control, since they would increase the chances of spread of the symbiont in the target host populations.

To verify the capability of Asaia to move from one host to another and efficiently colonize different body systems, we used three insect models having as common trait diets based on sugar-rich matrices. Two of the insect models are phylogenetically closely related, the mosquitoes An. stephensi and Ae. aegypti (Diptera). The third, the leafhopper S. titanus (Hemiptera), is phylogenetically distant from the other two. Indeed, Diptera and Hemiptera represent lineages that likely separated over $\mathbf{3 0 0}$ million 
years ago (the holometabolous and heteromethabolous lineages respectively).

We successfully detected and isolated Asaia sp. from Ae. aegypti. This finding represents an original result if compared with previous studies investigating the microbiota of this insect (Luxananil et al., 2001; Gusmão et al., 2007). We speculate that the failure of previous studies in detecting and isolating this $A A B$ possibly derives from the primers used in PCR and the lack of a suitable cultivation method. Indeed, Asaia is a relatively slow growing bacterium, having a duplication time of about $2 \mathrm{~h}$. Hence, it can be easily overgrown by fast growing bacteria unless selection factors are used. To overcome this inconvenience we used a pre-enrichment step in a liquid medium at $\mathrm{pH} 3.5$ that has already been used for isolating Asaia from plant material (Yamada et al., 2000). Such a selection allows Asaia to overgrow acid-sensitive bacteria.

In S. titanus, we were able to detect Asaia in 30 of 40 individuals analysed. However, we cannot actually define if Asaia is fixed in the population of $S$. titanus since a higher number of individuals should be tested. We could not isolate Asaia in pure culture from any of the $S$. titanus individuals tested. The lower prevalence of Asaia in $S$. titanus than in mosquitoes as detected by PCR, suggests that $S$. titanus could be a niche supporting Asaia growth, however, less favourable than mosquitoes of the Anopheles or Aedes genera. This is in agreement with Ashbolt and Inkerman (1990), who found that the presence of $A A B$ associated with leafhoppers of the species Perkinsiella saccharicida was low ( $10^{3} \mathrm{AAB}$ per individual) compared with that determined on the pink sugar cane mealybug Saccharococcus sacchari $\left(10^{6} \mathrm{AAB}\right.$ per individual). On the other hand, the high number of Asaia cells detected by quantitative PCR in some $S$. titanus individuals could be due to an actively feeding state of the insect, as they were maintained for some days on sucrose solution. In our experiments, we did not attempt the isolation of Asaia from individuals fed with artificial sucrose solution in the laboratory. Another explanation of the failure in isolating Asaia from $S$. titanus is that the symbiont of the leafhopper could have different nutritional requirements from those of mosquitoes, driven by the different type of diet of the host. To verify this hypothesis further media and cultivation conditions different from those we adopted should be used.

The presence of $A A B$ in insects has been documented since 1981 (Lambert et al., 1981). In the last few years an increased number of studies reported $A A B$ as a remarkable component of the microbial community associated to arthropods, raising the question of the role of these microorganisms in the animal host. There is an apparent correlation between $A A B$ and the insect nutritional behaviour. Acetic acid bacteria have been found associated with insects with a sugar-based diet. Indeed, AAB are well known to grow on a wide range of sugars with differences according to their metabolisms, e.g. Gluconobacter spp. prefer sugar-rich niches, while Acetobacter spp. predominate in ethanol-containing environment. Among $A A B$, Asaia spp. show unique characteristics: no or scarce production of acetic acid from ethanol, inhibition of growth in presence of $0.35 \%$ acetic acid and weak capability of oxidizing acetate to carbon dioxide and water (Kersters et al., 2006; Ano et al., 2008). We identified Asaia in Ae. aegypti adults, which feed on nectar and other sugar solutions. According to plant species, nectar contains sucrose, glucose and fructose. The leafhopper $S$. titanus feeds on the phloem sap that contains sucrose as a main carbon substrate. The relationship between a sugarfeeding habit of the insect host and the presence of $A A B$ in the body has been found in mosquitoes and leafhoppers, but also in other insects feeding on sugar-rich matrices, like A. mellifera, D. melanogaster and S. sacchari (Ashbolt and Inkerman, 1990; Jeyaprakash et al., 2003; Mohr and Tebbe, 2006; Babendreier et al., 2007; CorbyHarris et al., 2007; Cox and Gilmore, 2007; Ren et al., 2007; Ryu et al., 2008).

The characteristics of the feeding material combined with those of the external environment are also important in supporting $A A B$ presence and favouring their diffusion. Nectar has a low pH (3.9) (Mohr and Tebbe, 2006 and references therein), the exudate of mealybugs is acidic $(\mathrm{pH} 3)$ with fructose as the major sugar present (Ashbolt and Inkerman, 1990) and the surface of Drosophila spp. can be contaminated by ethanol (Ren et al., 2007). Similarly to the external surface, the insect gut represents a unique environment that favours the survival of specific bacterial species (Ren et al., 2007), as it occurs in vertebrates. It is generally assumed that the survival of microorganisms in the gut requires resistance to $\mathrm{pH}$, redox potential and ionic strength of the midgut and to the digestive enzymes. For example, the acidity of the crop and the hindgut (Dow and Harvey, 1988; Moffett and Koch, 1992; Clark, 1999), like the low $\mathrm{pH}$ in the gut of $A$. mellifera (Mohr and Tebbe, 2006 and references therein), likely contributes to support the proliferation of $A A B$ in these insects. In Anopheles spp., the presence of a polysaccharidic extracellular matrix around the cells of Asaia (Favia et al., 2007) could protect the bacterium from adverse conditions, such as alkaline or acidic $\mathrm{pH}$, like that of larvae (Dadd, 1975; Corena et al., 2005) or of adults (Gusmão et al., 2007), respectively, or high osmolarity (Yukphan et al., 2005).

Whatever it is the role of Asaia in the biology of the insects investigated (the documented presence in various species of mosquitoes, i.e. An. stephensi, An. maculipennis, An. gambiae, Ae. aegypti, would suggest a key role) its identification in species of different orders, like Diptera 
and Hemiptera, suggests that it may be a widespread symbiont of insects. The efficiency of interspecies transfection of bacterial symbionts has been previously demonstrated with horizontal colonization experiments of tsetse flies with bacterial cells of the genus Sodalis (Weiss et al., 2006). The data of colonization experiments with fluorescent proteins-expressing Asaia cells reported in the present study proved the capability of this bacterium to cross-colonize insects belonging to phylogenetically distant orders. In two phylogenetically related mosquito species, like An. stephensi and Ae. aegypti, a different body colonization prevalence by a bacterial strain isolated from the other mosquito species was observed. In all the organs examined Ae. aegypti colonized by Asaia strain SF2.1(Gfp) showed a remarkably lower percentage of infected individuals than An. stephensi colonized with Asaia strain AE10.8(Gfp). However, we cannot deduce from the actual experimental design if these differences were to be attributed to the insect host or the Asaia strain used. We speculate that different bacterial phenotypes, like cell surface properties, could remarkably affect the cross-colonization host range and capability. Indeed, the different colonization efficiency observed with the different host-symbiont combinations indicates that further research work should be done to understand the drivers of colonization specificity.

After acquisition with the diet, Asaia reaches the gut lumen and, crossing the numerous physical and biochemical barriers that prevent the entrance in the haemocoel of undesired microorganisms (Vallet-Gely et al., 2008), it is able to colonize different body parts. In this regard, when experiments of gene silencing were carried out on AgDscam, an essential factor of An. gambiae immune defence against bacteria, a remarkable proliferation of bacteria, including $A$. bogorensis, occurred within the mosquito haemolymph (Dong et al., 2006). Besides the guts of Ae. aegypti, An. stephensi and S. titanus, the Gfp-labelled strain showed also the ability to efficiently colonize the salivary glands and the male and female reproductive organs of Ae. aegypti and $S$. titanus, highlighting a colonization pattern similar to that previously reported for An. stephensi (Favia et al., 2007). As already proved for An. stephensi, the abundance of Asaia in different tissues of the reproductive organs may indicate a possible transmission of the bacterium from the mother (Favia et al., 2007) or the father (Damiani et al., 2008) to the offspring. It is noteworthy the detection of Asaia by $\mathrm{FISH}$ all around ovarian eggs of $S$. titanus (Fig. 2D-F) as well as the finding of Gfp-labelled Asaia around immature eggs of this insect (Figs $2 \mathrm{H}$ and 4D). We could thus hypothesize that vertical transmission of Asaia in S. titanus occurs through egg smearing and/or by the penetration of the bacteria across the egg membrane during the egg development.
Although the mechanism of the vertical transmission route remains to be elucidated, the capability of the bacterium to be widespread in natural insect populations represents an important property for its application in potential symbiotic control strategies both as a natural and as a paratransgenic delivery system (Riehle and Jacobs-Lorena, 2005). Understanding the nature of the symbiosis supported by Asaia in the different hosts, its role for the insect biology and some details of the vertical transmission are important issues that remain to be answered. However, the capability of the bacterium to be widespread in different and phylogenetically distant insect species, together with the easy culturability, transformability and the efficiency in colonizing different hosts, makes this bacterium an interesting candidate for symbiotic control of human diseases, such as malaria or dengue fever, as well as plant diseases like the Flavescence Dorée of grapevine (Marzorati et al., 2006).

\section{Experimental procedures}

\section{Insect collection}

Aedes aegypti individuals came from a lab strain kindly provided by the Suisse Tropical Institute (Basilea) and reared in the laboratory at the University of Camerino (Italy) since 2006. Anopheles stephensi samples came from a colony reared since 1988 at the University of Camerino. Mosquitoes were kept in cages $(25 \times 25 \times 25 \mathrm{~cm})$ made of a steel frame covered with nettings and maintained at $30 \pm 1^{\circ} \mathrm{C}$ temperature, $70 \pm 5 \%$ humidity, with a dark/light period of $12 \mathrm{~h}$. Scaphoideus titanus individuals were collected in vineyards with heavy symptoms of Flavescence Dorée from various areas of the Piedmont region during the summer season. Field-collected individuals were immediately used for Asaia isolation. For quantitative real-time PCR experiments, $S$. titanus individuals were kept for a week in laboratory cages at the University of Turin (Italy) and maintained on standard, sterilized sucrose solution at room temperature $\left(25-30^{\circ} \mathrm{C}\right)$, before killing for the subsequent analyses.

\section{Transmission electron microscopy}

Laboratory-reared Ae. aegypti and field-collected S. titanus insects were dissected with sterile scalpels and little pliers in a sterile saline solution to separate the salivary glands, the gut, the fat bodies and the ovaries. Samples were fixed in $0.1 \mathrm{M}$ cacodylate buffer ( $\mathrm{pH} 7.2$ ) containing $2.5 \%$ glutaraldehyde for $3 \mathrm{~h}$ at $4^{\circ} \mathrm{C}$. The samples were then washed in the same buffer and post-fixed in $1 \% \mathrm{OsO}_{4}$ in the same buffer for $1.5 \mathrm{~h}$ at $4^{\circ} \mathrm{C}$. Successively, all samples were dehydrated in ethanol and embedded in Epon 812 . Thin sections $(80 \mathrm{~nm})$ were stained with uranil acetate and lead citrate and examined under a Zeiss EM900 transmission electron microscope.

\section{DNA extraction and PCR-based analyses}

DNA extraction from whole $S$. titanus and Ae. aegypti individuals and from dissected organs of Ae. aegypti was 
performed as previously described (Favia et al., 1994). Extracted DNA was used as template in conventional PCRs with Asaia-specific primers, Asafor (5'-GCG CGT AGG CGG TTT ACA C-3') and Asarev (5'-AGC GTC AGT AAT GAG CCA GGT T-3'), targeted on the 16S rRNA gene of this bacterium. Reaction mixtures were carried out in a final volume of $25 \mu \mathrm{l}$, using 0.2 units of Taq Gold DNA polymerase (Roche), 1× PCR buffer, $2.5 \mathrm{mM}$ of each dNTP, $25 \mathrm{pmol}$ of each primer, $2 \mathrm{mM} \mathrm{MgCl} 2$ and $50 \mathrm{ng}$ of DNA. Reactions were run for $10 \mathrm{~min}$ at $94^{\circ} \mathrm{C}$, followed by 30 cycles of $30 \mathrm{~s}$ at $94^{\circ} \mathrm{C}, 30 \mathrm{~s}$ at $60^{\circ} \mathrm{C}, 45 \mathrm{~s}$ at $72^{\circ} \mathrm{C}$ and then a final extension of $10 \mathrm{~min}$ at $72^{\circ} \mathrm{C}$. The $180 \mathrm{bp}$ PCR products were randomly chosen and sequenced with Asafor primer. Sequences were compared with the sequence database at the National Centre for Biotechnology Information by using BLASTN (Altschul et al., 1990).

Quantitative real-time PCRs were performed on the I-Cycler (Bio-Rad) using Asaia-specific primer set Asafor/ Asarev or eubacterial universal primers 357F (5'-CTA CGG GAG GCA GCA G-3') and 907R (5'-CCG TCA ATT CCT TTG AGT TT-3') according to Favia and colleagues (2007). For realizing standard curves, the 16S rRNA gene of Asaia amplified by PCR was cloned using pGEM T-easy Vector Cloning Kit (Promega). Following calculation of 16S rRNA gene copies of Bacteria and Asaia, the Asaia to Bacteria 16S rRNA gene copy ratio (ABR) was calculated and used as an estimate of the relative abundance of Asaia sp. in the bacterial community associated with different individuals of $S$. titanus (Favia et al., 2007).

\section{In situ hybridization and whole mount fluorescent in situ hybridization}

Field-collected $S$. titanus insects were dissected in sterile saline. ISH on paraffin-embedded sections of organs and tissues was performed as described previously (Beninati et al., 2004; Favia et al., 2007). Briefly, formamide concentration was adjusted at $30 \%$ and hybridization temperature was set at $46^{\circ} \mathrm{C}$. For specific detection of Asaia cells, two probes, Asaia1 (5'-AGC ACC AGT TTC CCG ATG TTA T-3') and Asaia2 (5'-GAA ATA CCC ATC TCT GGA TA-3'), were used as described previously (Favia et al., 2007). Probe EUB338, routinely used as a universal bacterial probe even though several phyla are not completely covered (Daims et al., 1999), was used as a bacterial positive control. Probes Asaia1 and Asaia2 have the following mismatches with the next non-target $A A B$ (in parentheses are reported the 16S rRNA gene accession number and the numbers of mismatches for probe Asaia1 and Asaia2 respectively): Halobharath swaminathanianus (AF459454, 1,1); Kozakia baliensis (AB056321, 2,2); Gluconacetobacter hansenii (X75620, 4,2); Gluconacetobacter kombuchae (AY688433, $4,2)$; Gluconobacter oxydans (AB308275, 3,6); Gluconobacter japonicus (AB470922, 4,3); Acetobacter tropicalis (AB470916, 4,2); Acetobacter syzygii (AB264094, 4,2). The probes were $5^{\prime}$-labelled with digoxigenin in order to be recognized by an antidigoxigenin antibody coupled with a horseradish peroxidase. Staining was performed with 3-amino-9ethylcarbazole and observation was with a light microscope (Favia et al., 2007). Alternatively, for fluorescent in situ hybridization (FISH) probes Asaia1 and Asaia2 were labelled at the $5^{\prime}$ end with the fluorochrome Cy5 (indodicarbocyanine, absorption/emission at $650 / 670 \mathrm{~nm}$ ) or with the fluorochrome Сy3 (indocarbocyanine, absorption/emission at 550/570 nm). The probe EUB338 were labelled with fluorescein isothiocyanate (FITC, absorption/emission at 494/520 nm). Insect dissections were fixed in a $4 \%$ solution of paraformaldehyde in PBS $1 \times$ for $30 \mathrm{~min}$ and then washed in PBS 1×. Samples were incubated for $1 \mathrm{~min}$ at the temperature of $37^{\circ} \mathrm{C}$ with a $100 \mu \mathrm{g} \mathrm{ml}^{-1}$ pepsin solution and washed again in PBS $1 \times$. Successively, an overnight hybridization was carried out in dark conditions with $50 \mu \mathrm{l}$ of hybridization buffer (2× SSC, $50 \%$ formamide, $1 \mu \mathrm{g} \mathrm{ml}^{-1}$ probes). Dissections were then washed in $500 \mu$ l of washing buffer (2x SSC and $50 \%$ formamide) and subsequently two washes in $0.1 \times \mathrm{SSC}$ at $37^{\circ} \mathrm{C}$ were performed, followed by two washes in $1 \times \mathrm{PBS}$ at $37^{\circ} \mathrm{C}$. Then $50 \mathrm{ng}$ of propidium iodide or DAPI were added, and incubated for $5 \mathrm{~min}$ at room temperature. After two washes in $1 \times$ PBS at $37^{\circ} \mathrm{C}$, samples were mounted in antifading medium, then observed in a laser-scanning confocal microscope SP2-AOBS (Leica).

Both for ISH and FISH, control experiments involved either treatment of slides with RNase prior to probe hybridization step or the absence of the probe. Positive control experiments with the universal bacterial probe EUB338 (Fuchs et al., 1998) were also performed applying the same conditions.

\section{Asaia $s p$. isolation}

Asaia strains were isolated from four adult mosquitoes using an enrichment medium ( $\mathrm{pH}$ 3.5) as described by Yamada and colleagues (2000). Mosquitoes were washed three times with $0.9 \% \mathrm{NaCl}$ and homogenated in $200 \mu \mathrm{l} 0.9 \% \mathrm{NaCl}$. Twenty microlitres of homogenate were inoculated into the enrichment medium and let to grow at $30^{\circ} \mathrm{C}$ for 2 days with shaking (160 r.p.m.). When microbial growth occurred, the microorganisms were plated on calcium carbonate agar plates. Circular, pink-pigmented colonies capable of causing clearing of the calcium carbonate were selected. The identification as Asaia sp. was confirmed by specific Asaia PCR amplification with Asafor and Asarev primers and by sequencing of the almost entire 16S rRNA gene after amplification with universal bacterial primers 27F (5'-TCG ACA TCG TTT ACG GCG TG-3') and 1495R (5'-CTA CGG CTA CCT TGT TAC GA-3').

For isolation of Asaia from $S$. titanus the same procedure for isolation from Ae. aegypti was adopted, except that, besides sorbitol, other different carbon sources, including glucose, sucrose, mannitol, were used in single or in mixture in the pre-enrichment step. A total of 40 individuals recovered from different vineyard in Piedmont were homogenated and used for isolation.

\section{Construction of Asaia tagged with fluorescent proteins}

Two transformants of Asaia strain SF2.1, isolated from An. stephensi, and one transformant of strain AE10.8, isolated from Ae. aegypti, were used in colonization experiments of the insects. Strain SF2.1(Gfp) was prepared as previously described (Favia et al., 2007). This strain was tagged with plasmid pHM2-Gfp and used in feeding 
experiments in presence of $100 \mu \mathrm{ml}^{-1}$ kanamycin. Strain AE10.8(Gfp) was prepared by the same procedure used for strain SF2.1.

Strain SF2.1(DsRed) (Damiani et al., 2008) was constructed by insertion of a mini-Tn5 gene cassette containing the dsRed gene expressed from E. coli ribosomal promoter rrnBP1 into the chromosome of Asaia SF2.1 by conjugation and transposition (Mølbak et al., 2007). Briefly, the delivery plasmid TTN151 was mobilized from E. coli Mv1190גpir to the recipient using the helper strain E. coli HB101(pRK600). Transconiugants that underwent transposition events were selected by plating on GLY medium at $\mathrm{pH} 4.0$ (where E. coli cannot grow) supplemented with kanamycin $\left(200 \mu \mathrm{g} \mathrm{ml}^{-1}\right)$. DsRed fluorescence of colonies was observed using a Leica DM4000B equipped with Leica filter set $G / R$ (excitation filter, BP 490/20 nm; dichromatic mirror, 505; emission filter, LP $525 / 20$ and excitation filter, BP $575 / 30 \mathrm{~nm}$; dichromatic mirror, 600; emission filter, LP 635/40). Among the Asaia transconjugants, a clone which grew at the same rate as SF2.1, named strain SF2.1(DsRed), was selected for performing recolonization experiments in $A$. aegypti and $S$. titanus without the presence of antibiotics in the feeding solution.

\section{Evaluation of stability of Asaia strains tagged with fluorescence proteins}

Asaia SF2.1(Gfp) was grown overnight in GLY medium with kanamycin $\left(100 \mathrm{~g} \mathrm{~m} \mathrm{ml}^{-1}\right)$ and diluted the next day into fresh GLY medium without antibiotic. The culture was allowed to grow to an $\mathrm{OD}_{600}$ of $\sim 1$ and then diluted. This was repeated three consecutive times over the course of experiment in order to follow the bacterium for a total of approximately 36 generations. The proportion of kanamycin-resistant bacterial cells was determined by plating on GLY agar plates added with kanamycin every time that the culture reached an $\mathrm{OD}_{600}$ of 1 . The total cells were in parallel counted by plating on GLY plates without the antibiotic. The same procedure was used for strains AE10.8(Gfp) and SF2.1(DsRed).

\section{Colonization of insects by Asaia expressing Gfp and DsRed}

Asaia strains SF2.1(Gfp), AE 10.8(Gfp) and SF2.1(DsRed) were used in colonization experiments of An. stephensi, $A e$. aegypti and $S$. titanus. The first two strains were always used under the selection of kanamycin, while the third strain was used without antibiotic. Strain SF2.1(Gfp) was used in colonization experiments of Ae. aegypti, while strain AE10.8(Gfp) was used for the colonization of An. stephensi. In the case of $S$. titanus leafhoppers, they were fed with either SF2.1(DsRed) or strain SF2.1(Gfp). After growing at $30^{\circ} \mathrm{C}$ cells were harvested by centrifugation (10 min, $3000 \mathrm{~g}$ ), washed three times with $0.9 \% \mathrm{NaCl}$ and adjusted to $10^{8}$ cells $\mathrm{ml}^{-1}$ in $30 \mathrm{ml}$ of $5 \%(\mathrm{w} / \mathrm{v})$ sucrose aqueous solution for An. stephensi and Ae. aegypti, or $5 \%$ sucrose solution in Tris-EDTA pH 8 for $S$. titanus. Cell suspensions, supplemented with $100 \mu \mathrm{g} \mathrm{ml}^{-1}$ of kanamycin for strains SF2.1(Gfp) and $A E 10.8(G f p)$, were administrated to the insects allowing them to feed for periods of 2 days after which the bacterial feeding solutions were substituted with equivalent, cell-free solutions with the addition of the kanamycin when foreseen. After 12, 24, 48 and $72 \mathrm{~h}$ after exposure to the cells suspensions, the insects were withdrawn and gut, salivary glands and reproductive organs were dissected in PBS. The organs were fixed with $4 \%$ paraformaldeyde for $10 \mathrm{~min}$ at $4^{\circ} \mathrm{C}$ with the exception of the salivary glands. The slides were then mounted in glycerol-PBS for the microscopy analyses. The samples were analysed with IX71 fluorescence microscope (Olympus, Melville, NY, USA) and a MRC600 confocal laser scanning microscope (Bio-Rad).

As colonization controls, two bacteria never associated to insects has been used. The first was a strain of $P$. putida, a typical inhabitant of soil. The second was E. coli a typical symbiont of the gut of mammals. For the mosquitoes, An. stephensi and Ae. aegypti, Asaia SF2.1(Gfp) and E. coli $\mathrm{DH} 5 \alpha$ pKan(DsRed) were used for cocolonization experiments. Both bacteria carried a plasmid with a gene cassette encoding a fluorescent protein (Gfp in the case of Asaia, DsRed in the case of E. coli). In the case of $S$. titanus, Pseudomonas putida KT2442 (gfp Rif') and E. coli DH5 $\alpha$ pKan(DsRed) were independently used alone, for colonization experiments, or in combination with Asaia SF2.1(DsRed) and Asaia SF2.1(Gfp), respectively, for cocolonization experiments. P. putida KT2442 (gfp Rifr) carried a Gfp cassette in

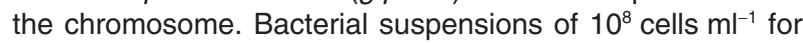
each strain were appropriately prepared and administered to the insects as described above. The samples were then processed, as already indicated.

\section{Sequence accession numbers}

Sequences of Asaia strains AE5.2, AE6.5 and AE10.8 isolated from Ae. aegypti and of two Asaia-specific PCR fragments amplified from the metagenome of Ae. aegypti and S. titanus (named AE4 and ST2 respectively) have been deposited in the DDBJ-EMBL-GenBank databases under the accession numbers FN297840-FN297844.

\section{Acknowledgements}

We thank K. J. Heller (Institute for Microbiology, Federal Dairy Research Center for Nutrition and Food, Kiel, Germany) for providing us plasmids pHM2 for transformation experiments of Asaia sp., and Dr L. J. Halverson (lowa State University, Ames, IA, USA) for the gfp gene cassette, used for construction of the Gfp-tagged Asaia strains, and N. Kroer (Department of Environmental Chemistry and Microbiology, National Environmental Research Institute, Lyngby, Denmark) for providing plasmids for the construction of Asaia strains labelled with DsRed. We thank K. Smalla (BBA Institute for Plant Virology, Microbiology and Biosafety, Brunswick, Germany) for providing P. putida KT2442 ( $g f p$ Rifr). Partial financial contribution comes form the Italian Ministry for Research (MIUR), in the ambit of projects PRIN 2007 'Caratterizzazione del microbiota associato a Scaphoideus titanus e Hyalesthes obsoletus, cicaline vettrici di fitoplasmi nella vite ed isolamento e studio della localizzazione di batteri acetici simbionti'. E.C., P.R., C.B and D.D. benefited of travel grants from Cost Action FA0701: 'Arthropod Symbiosis: From Fundamental Studies to Pest and Disease Management'. N.R. 
was partially supported by the Università degli Studi di Torino and the Regione Piemonte in the ambit of the project 'Studio dei microrganismi simbionti di artropodi vettori di patologie umane e vegetali e di nuove strategie di Controllo Simbiotico della trasmissione' (assegno cofinanziato dalla Regione Piemonte - Rep. N. 12607 del 30/07/2007).

\section{References}

Altschul, S.F., Gish, W., Miller, W., Myers, E.W., and Lipman, D.J. (1990) Basic local alignment search tool. J Mol Biol 215: 403-410.

Ano, Y., Toyama, H., Adachi, O., and Matsushita, K. (2008) Energy metabolism of a unique acetic acid bacterium, Asaia bogorensis, that lacks ethanol oxidation activity. Biosci Biotechnol Biochem 72: 989-997.

Ashbolt, N.J., and Inkerman, P.A. (1990) Acetic acid bacterial biota of the pink sugar cane mealybug, Saccharococcus sacchari, and its environs. Appl Environ Microbiol 56: 707712.

Babendreier, D., Joller, D., Romeis, J., Bigler, F., and Widmer, F. (2007) Bacterial community structures in honeybee intestines and their response to two insecticidal proteins. FEMS Microbiol Ecol 59: 600-610.

Beninati, T., Lo, N., Sacchi, L., Genchi, C., Noda, H., and Bandi, C. (2004) A novel alpha-Proteobacterium resides in the mitochondria of ovarian cells of the tick Ixodes ricinus. Appl Environ Microbiol 70: 2596-2602.

Clark, T.M. (1999) Evolution and adaptive significance of larval midgut alkalinization in the insect superorder. J Chem Ecol 25: 1945-1960.

Corby-Harris, V., Pontaroli, A.C., Shimkets, L.J., Bennetzen, J.L., Habel, K.E., and Promislow, D.E.L. (2007) Geographical distribution and diversity of bacteria associated with natural populations of Drosophila melanogaster. Appl Environ Microbiol 73: 3470-3479.

Corena, M.D., VanEkeris, L., Salazar, M.I., Bowers, D., Fiedler, M.M., Silverman, D., et al. (2005) Carbonic anhydrase in the adult mosquito midgut. J Exp Biol 208: 3263-3273.

Cox, C.R., and Gilmore, M.S. (2007) Native microbial colonization of Drosophila melanogaster and its use as a model of Enterococcus faecalis pathogenesis. Infect Immun 75: 1565-1576.

Dadd, R.H. (1975) Alcalinity within the midgut of mosquitos larvae with alkaline-active digestive enzymes. J Insect Physiol 21: 1847-1853.

Daims, H., Brühl, A., Amann, R., Schleifer, K.H., and Wagner, M. (1999) The domain-specific probe EUB338 is insufficient for the detection of all Bacteria: development and evaluation of a more comprehensive probe set. Syst Appl Microbiol 22: 434-444.

Dale, C., and Moran, N. (2006) Molecular interaction between bacterial symbionts and their hosts. Cell 126: 453-465.

Damiani, C., Ricci, I., Crotti, E., Rossi, P., Rizzi, A., Scuppa, P., et al. (2008) Paternal transmission of symbiotic bacteria in malaria vectors. Curr Biol 18: R1087-R1088.

Dong, Y., Taylor, H.E., and Dimopoulos, G. (2006) AgDscam, a hypervariable immunoglobulin domain-containing receptor of the Anopheles gambiae innate immune system. PLoS Biol 4: 1138-1146.

Dow, J.A., and Harvey, W.R. (1988) Role of midgut electrogenic $\mathrm{K}^{+}$pump potential difference in regulating lumen $\mathrm{K}^{+}$and $\mathrm{pH}$ in larval lepidoptera. J Exp Biol 140: 455463.

Favia, G., Dimopoulos, G., della Torre, A., Touré, Y.T., Coluzzi, M., and Louis, C. (1994) Polymorphisms detected by random PCR distinguish between different chromosomal forms of Anopheles gambiae. Proc Natl Acad Sci USA 91: 10315-10319.

Favia, G., Ricci, I., Damiani, C., Raddadi, N., Crotti, E., Marzorati, M., et al. (2007) Bacteria of the genus Asaia stably associate with Anopheles stephensi, an Asian malarial mosquito vector. Proc Natl Acad Sci USA 104: 9047-9051.

Feldhaar, H., and Gross, R. (2009) Insects as hosts for mutualistic bacteria. Int $J$ Med Microbiol 299: 1-8.

Fuchs, B.M., Wallner, G., Beisker, W., Schwippl, I., Ludwig, W., and Amann, R. (1998) Flow cytometric analysis of the in situ accessibility of Escherichia coli 16S rRNA for fluorescently labeled oligonucleotide probes. Appl Environ Microbiol 42: 4973-4982.

Gusmão, D.S., Santos, A.D., Marini, D.C., de Souza, E., Dias Peixoto, A.M., Bacci Júnior, M., et al. (2007) First isolation of microorganisms from the gut diverticulum of Aedes aegypti (Diptera: Culicidae): new perspectives for an insect-bacteria association. Mem Inst Oswaldo Cruz 102: 919-924.

Jeyaprakash, A., Hoy, M.A., and Allsopp, M.H. (2003) Bacterial diversity in worker adults of Apis mellifera capensis and Apis mellifera scutellata (Insecta: Hymenoptera) assessed using $16 S$ rRNA sequences. J Invertebr Pathol 84: 96-103.

Katsura, K., Kawasaki, H., Potacharoen, W., Saono, S., Seki, T., Yamada, Y., et al. (2001) Asaia siamensis sp. nov., an acetic acid bacterium in the alfa-Proteobacteria. Int $J$ Syst Evol Microbiol 51: 559-563.

Kersters, K., Lisdiyanti, P., Komagata, K., and Swings, J. (2006) The family Acetobacteraceae: the genera Acetobacter, Acidomonas, Asaia, Gluconacetobacter, Gluconobacter, and Kozakia. In The Prokaryotes, Vol. 5. Dworkin, M., Falkow, S., Rosenberg, E., Schleifer, K.-H., Stackebrandt, E. (eds). New York, USA: Springer, pp. 163-200.

Klappenbach, J.A., Saxman, P.R., Cole, J.R., and Schmidt, T.M. (2001) rrnDB: the ribosomal RNA operon copy number database. Nucleic Acids Res 29: 181-184.

Kommanee, J., Akaracharanya, A., Tanasupawat, S., Malimas, T., Yukphan, P., Nakagawa, Y., and Yamada, Y. (2008) Identification of Acetobacter strains isolated in Thailand based on 16S-23S rRNA gene ITS restriction and 16S rRNA gene sequence analyses. Ann Microbiol 58: 319324.

Kounatidis, I., Crotti, E., Sapountzis, P., Sacchi, L., Rizzi, A., Chouaia, B., et al. (2009) Acetobacter tropicalis is a major symbiont in the olive fruit fly Bactrocera oleae. Appl Environ Microbiol 75: 3281-3288.

Lambert, B., Kersters, K., Gosselé, F., Swings, J., and De Ley, J. (1981) Gluconoacetobacters from honey bees. Antonie van Leeuwenhoek. J Microbiol Serol 47: 147157.

Luxananil, P., Atomi, H., Panyim, S., and Imanaka, T. (2001) 
Isolation of bacterial strains colonisable in mosquito larval guts as novel host cells for mosquito control. J Biosci Bioeng 92: 342-345.

Malimas, T., Yukphan, P., Takahashi, M., Kaneyasu, M., Potacharoen, W., Tanasupawat, S., et al. (2008) Asaia lannaensis sp. nov., a new acetic acid bacterium in the Alphaproteobacteria. Biosci Biotechnol Biochem 72: 666671.

Marzorati, M., Alma, A., Sacchi, L., Pajoro, M., Palermo, S., Brusetti, L., et al. (2006) A novel Bacteroidetes symbiont is localized in Scaphoideus titanus, the insect vector of Flavescence Dorée in Vitis vinifera. Appl Environ Microbiol 72: 1467-1475.

Moffett, D.F., and Koch, A. (1992) Driving forces and pathways for $\mathrm{H}^{+}$and $\mathrm{K}^{+}$transport in insect midgut goblet cells. $J$ Exp Biol 172: 403-415.

Mohr, K.I., and Tebbe, C.C. (2006) Diversity and phylotype consistency of bacteria in the guts of three bee species (Apoidea) at an oilseed rape field. Environ Microbiol 8: 258-272.

Mølbak, L., Molin, S., and Kroer, N. (2007) Root growth and exudate production define the frequency of horizontal plasmid transfer in the Rhizosphere. FEMS Microbiol Ecol 59: 167-176.

Moran, N.A. (2006) Symbiosis. Curr Biol 16: R866-R871.

Ren, C., Webster, P., Finkel, S.E., and Tower, J. (2007) Increased internal and external bacterial load during Drosophila aging without life-span trade off. Cell Metab 6: 144-152.

Riehle, M.A., and Jacobs-Lorena, M. (2005) Using bacteria to express and display anti-parasite molecules in mosquitos: current and future strategies. Insect Biochem Mol Biol 35: 699-707.

Ryu, J.-H., Kim, S.-H., Lee, H.-Y., Bai, J.Y., Nam, Y.-D., Bae, J.-W., et al. (2008) Innate immune homeostasis by the homeobox gene Caudal and commensal-gut mutualism in Drosophila. Science 319: 777-782.

Schaechter, M., Ingraham, J.L., and Neidhardt, F.C. (2006) Microbe. Washington, DC, USA: ASM Press, p. 46.

Vallet-Gely, I., Lemaitre, B., and Boccard, F. (2008) Bacterial strategies to overcome insect defences. Nat Rev Microbiol 6: 302-313.

Weiss, B.L., Mouchotte, R., Rio, R.V.M., Wu, Y., Wu, Z., Heddi, A., and Aksoy, S. (2006) Interspecific transfer of bacterial endosymbionts between tseyse fly species: infection establishment and effect on host fitness. Appl Environ Microbiol 72: 7013-7021.

Yamada, Y., Katsura, K., Kawasaki, H., Widyastuti, Y., Saono, S., Seki, T., et al. (2000) Asaia bogorensis gen. nov., sp. nov., an unusual acetic acid bacterium in the alfa-Proteobacteria. Int J Syst Evol Microbiol 50: 823-829.

Yukphan, P., Potacharoen, W., Tanasupawat, S., Tanticharoen, M., and Yamada, Y. (2004) Asaia krungthepensis sp. nov., an acetic acid bacterium in the alfaProteobacteria. Int J Syst Evol Microbiol 54: 313-316.

Yukphan, P., Malimas, T., Potacharoen, W., Tanasupawat, S., Tanticharoen, M., and Yamada, Y. (2005) Neoasaia chiangmaiensis gen. nov., sp. nov., a novel osmotolerant acetic acid bacterium in the $\alpha$-Proteobacteria. J Gen Appl Microbiol 51: 301-311. 